\title{
On the Elementary Mechanical Effects of the Space-Time-Symmetry Relativity
}

\author{
Mikhail Petelin' ${ }^{1}$ Manfred Thumm² \\ ${ }^{1}$ Institute of Applied Physics, Russian Academy of Sciences, Nizhny Novgorod, Russia; ${ }^{2}$ Institute for Pulsed Power \\ and Microwave Technology, Karlsruhe Institute of Technology, Karlsruhe, Germany
}

Correspondence to: Mikhail Petelin, petelin@ipfran.ru; Manfred Thumm, manfred.thumm@kit.edu

Keywords: Absolute Speed Limit, Restless and Unhurried Elementary Particles, Particle Wave Packets, Mass-Momentum Relation

Received: July 29, 2020 Accepted: September 24, 2020 Published: September 27, 2020

Copyright $\odot 2020$ by author(s) and Scientific Research Publishing Inc.

This work is licensed under the Creative Commons Attribution International License (CC BY 4.0).

http://creativecommons.org/licenses/by/4.0/

\section{(c) (i) Open Access}

\section{ABSTRACT}

This paper is an addendum to the article "On the Evolution of Approaches to the SpaceTime Symmetry", Natural Science, Vol. 10, No. 3, pp. 81-84, 2018. The analysis is based on the Galileo's postulate that all inertial reference frames are equal. The postulate results immediately in the absolute speed limit, which is identical for all bodies in all reference frames. Two kinds of elementary particles are admitted: unhurried and restless ones (exemplified, accordingly, with electrons and photons). Any particle may be treated as wave packet, the particle mass and momentum depending on the reference frame. Thus, space-time-symmetry is the natural background for the relativistic and quantum theories.

\section{GALILEO'S REFERENCE FRAMES}

Our methodical paper [1] was stimulated by the assumption of Refs. [2,3] that the number of independent relativistic postulates $[4,5]$ might be minimized. This economic approach leads to the following simple corollaries.

Figure 1 shows the famous Galileo's (anti-Aristotle) model of inertial reference frames: two boats moving on a lake [6]. Each of these frames carries its own coordinate-time system $\xi, \tau$ or $\xi^{\prime}, \tau^{\prime}$. The measurement units being taken identical, the symmetry-reciprocity of the reference frames is met with the following equations [1]

$$
\begin{aligned}
& \xi=\gamma \xi^{\prime}+\kappa \tau^{\prime} \\
& \tau=\kappa \xi^{\prime}+\gamma \tau^{\prime}
\end{aligned} \Leftrightarrow \begin{aligned}
& \xi^{\prime}=\gamma \xi-\kappa \tau \\
& \tau^{\prime}=-\kappa \xi+\gamma \tau
\end{aligned}
$$

being left-right cyclic recurrent due to the interrelation between the constant coefficients $\gamma$ and $\kappa$

$$
\gamma^{2}-\kappa^{2}=1
$$


Under the following Minkowski change of variables [4, 5]

$$
\hat{\tau}=\mathrm{i} \tau, \quad \hat{\tau}^{\prime}=\mathrm{i} \tau^{\prime}
$$

the transformation (1) becomes equivalent to the mutual rotation of Cartesian coordinates (Figure 2) [4, 7], and the cyclic recurrence condition (2) turns out to be equivalent to the Pythagoras theorem

$$
\sin ^{2} \alpha+\cos ^{2} \alpha=1 \text {. }
$$

According to (1), the relative velocity of the reference frames is

$$
\beta=\kappa / \gamma .
$$

Equation (4) and the Pythagoras condition (2), (2a) give

$$
\gamma=\frac{1}{\sqrt{1-\beta^{2}}} .
$$

The Galileo's space-time symmetry (1) and (2) results in the elementary kinematic effects $[4,5]$ : the "Lorentz length contraction" and the "paradox of twins".

\section{FREE BODY KINEMATICS}

According to (1), (2) $[1,4,5]$, if a body in Figure 1 moves with a velocity $\beta_{b}^{\prime}$ relative to the $\xi^{\prime}, \tau^{\prime}$ reference frame, the body velocity relative to the $\xi, \tau$ frame is

$$
\beta_{b}=\frac{\beta_{b}^{\prime}+\beta}{1+\beta \beta_{b}^{\prime}} .
$$

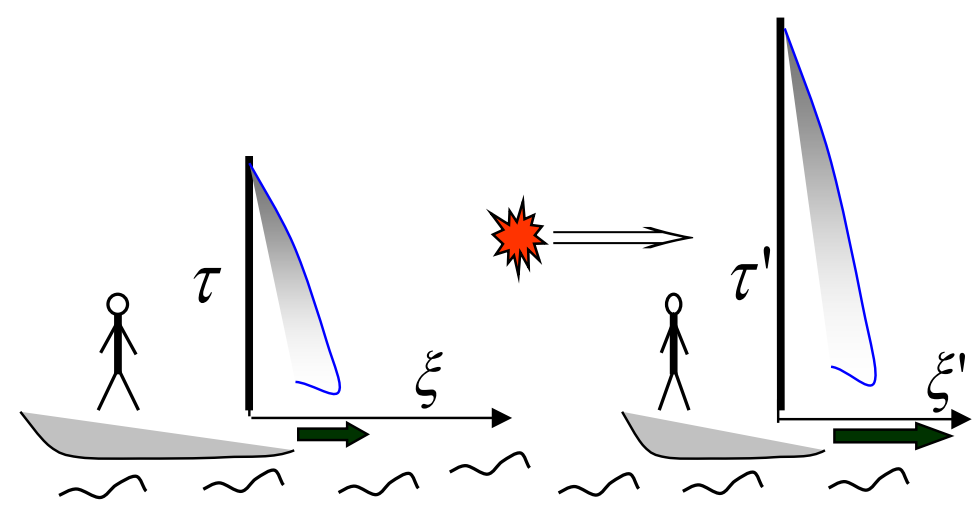

Figure 1. Galileo's inertial reference frames [1].

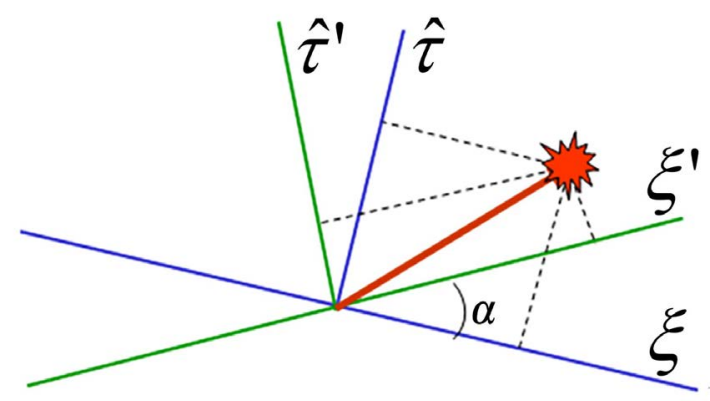

Figure 2. Mutual rotation of Cartesian coordinate systems [7] $\xi, \hat{\tau}$ and $\xi^{\prime}, \hat{\tau}^{\prime}$. 
It is natural to postulate that the body mass $m$ and the body momentum (quantity of body motion) $p$ compose a vector $[\hat{m}, p]$ similar to the time-coordinate vector $[\hat{\tau}, \xi]$ in the Minkowski scheme Figure 2 (as time may be arbitrary at zero coordinate, so mass may be arbitrary at zero momentum). Therefore, by analogy with the time-coordinate transformation (1), the mass-momentum transformation is

$$
\begin{aligned}
& p=\gamma p^{\prime}+\kappa m^{\prime} \\
& m=\kappa p^{\prime}+\gamma m^{\prime}
\end{aligned} \Leftrightarrow \begin{aligned}
& p^{\prime}=\gamma p-\kappa m \\
& m^{\prime}=-\kappa p+\gamma m
\end{aligned}
$$

The Galileo's space-time symmetry (1), (2) and (7) admits two kinds of elementary particles: restless and unhurried ones.

\subsection{Restless Particles}

According to the formulas (6) and (7), a particle may move in any reference frame with the ultimate velocity:

$$
\beta_{b}=\beta_{b}^{\prime}=1
$$

the particle mass and the particle momentum being interrelated with the formulas

$$
m=(\gamma+\kappa) m^{\prime}=(\gamma+\kappa) p^{\prime}=p \quad \Leftrightarrow \quad m^{\prime}=(\gamma-\kappa) m=(\gamma-\kappa) p=p^{\prime}
$$

(so the mass $=$ momentum is non-zero in any reference frame).

\subsection{Unhurried Particles}

If a body is resting in the reference frame $\xi^{\prime}, \tau^{\prime}$ :

$$
\beta_{b}^{\prime}=0, \quad p_{0}^{\prime}=0, \quad m^{\prime}=m_{0},
$$

then, according to (7), the mass and the momentum of the body in another reference frame $\xi, \tau$ are

$$
\begin{gathered}
m=\gamma m_{0} \\
p=\beta m .
\end{gathered}
$$

Therefore, as it follows from (11) and (5), if the body velocity $\beta_{b}$ approaches the universal speed limit, the body mass $m$ approaches infinity. For this reason, no real force can accelerate any unhurried particle up to the ultimate velocity equal to 1 .

\subsection{Common Speed Limitation}

According to (6)-(12), both restless and unhurried particles undergo the absolute speed limitation

$$
\beta_{b}, \beta_{b}^{\prime} \leq 1,
$$

corresponding to the $12^{\text {th }}$ Theorem of Proclus (proved on the contrary) [8]: "During a limited time it is not possible to go an infinite distance".

\section{WAVE APPROACH}

At the frame-to-frame transition described by (1), (2), and (7), and illustrated with Figure 2, the scalar product of the vectors $[\hat{m}, p]$ and $[\hat{\tau}, \xi]$ remains invariant:

$$
m \tau-p \xi=m^{\prime} \tau^{\prime}-p^{\prime} \xi^{\prime},
$$

which may be attributed to a phase of a relevant wave

$$
\mathrm{e}^{-i(m \tau-p \xi)}
$$

\subsection{Restless Particles}

If a particle is restless, according to (8) and (9), the wave (15) takes the form 


$$
\mathrm{e}^{-i m(\tau-\xi)}=\mathrm{e}^{-i p^{\prime}\left(\tau^{\prime}-\xi^{\prime}\right)},
$$

where the frequencies-propagators $m=p, m^{\prime}=p^{\prime}$ are interrelated with the formulas (9) corresponding to the Doppler effect [9]. The waves (16) may be composed in a packet

$$
\Phi(\tau-\xi)=\int_{-\infty}^{+\infty} g_{m} \mathrm{e}^{-i m(\tau-\xi)} \mathrm{d} m
$$

propagating with the ultimate speed equal to 1.

\subsection{Unhurried Particle}

If an unhurried particle, according to (10) - (12), is weakly relativistic (in a relevant reference frame)

$$
\kappa \ll 1 \text {, }
$$

the invariant (14) reduces to

$$
m \tau-p \xi=m_{0}(\gamma \tau-\kappa \xi) \approx m_{0} \tau+\theta_{\kappa}
$$

where

$$
\theta_{\kappa}=m_{0}\left(\frac{\kappa^{2}}{2} \tau-\kappa \xi\right) .
$$

The corresponding waves (15) of a common resting mass $m_{0}$ may be composed in a packet

$$
\Psi(\xi, \tau)=\int_{-\infty}^{+\infty} g_{\kappa} \mathrm{e}^{-i(m \tau-p \xi)} \mathrm{d} \kappa,
$$

which, with account of (19), may be written in the form

$$
\Psi \approx \psi \mathrm{e}^{-i m_{0} \tau},
$$

where the envelope

$$
\psi(\xi, \tau)=\int_{-\infty}^{+\infty} g_{\kappa} \mathrm{e}^{-i \theta_{k}} \mathrm{~d} \kappa
$$

slowly depends on time and coordinate. By using (20), one finds that $\psi(\xi, \tau)$ satisfies the following parabolic equation

$$
\frac{\partial \psi}{\partial \tau}=-\frac{i}{2 m_{0}} \frac{\partial^{2} \psi}{\partial \xi^{2}} .
$$

\section{CHANGE TO PRACTICAL SCALES}

The Galileo-symmetry-defined variables [used in the relations (1)] are matched to the ordinary space-time variables $x, t$ (used in the Lorentz transformation $[4,5]$ ) with the substitution [1]

$$
\begin{array}{lll}
\xi=x & \xi^{\prime}=x^{\prime} \\
\tau=c t & & \tau^{\prime}=c t
\end{array}
$$

where $c$ is the ultimate body velocity that should be measured in the usual units.

Relevant experiments were performed primarily with the light [9], which is an electromagnetic wave (16) composed of restless photons. The photon dimensional speed was measured to be $c=3 \times 10^{8} \mathrm{~m} / \mathrm{s}$ [9]. The wave (16) may be written in the form $\mathrm{e}^{-i(\omega t-k x)}$, where $\omega$ is the angular frequency and $k=\omega / c$ is the wave vector. In accordance to [5, 9], the "usual" photon energy is $\hbar \omega$ and the "usual" photon momentum is $\hbar k$, where $\hbar$ is the Planck constant divided by $2 \pi$. 
Results of subsequent experiments with unhurried charged particles (e.g. electrons) [10] proved to agree with the Galileo's space-time symmetry as well. The mass-momentum relations (11), (12) correspond with the famous Einstein's formula $E=M c^{2}[4,5,11]$ (Einstein in his original paper [11] uses $L$ for the particle energy $E$ and $V$ for the velocity of light $c$ ). At the coordinate-time change (25), if the "symmetric" resting mass $m_{0}$ and the "practical" mass $m_{e}$ of the electron are linearly interrelated $m_{0} \hbar=m_{e} c$, the electron wave-packet equation (24) may be converted to a form corresponding to the Schrödinger equation $[5,12]$.

\section{SUMMARY AND CONCLUSIONS}

The Galileo's space-time-symmetry postulate is followed by some mutually matched corollaries:

- the universal speed limit is equal for all material bodies in all inertial reference frames,

- both momentum and mass of any particle are different in different reference frames,

- $\quad$ any restless particle moves with the ultimate speed in any inertial reference frame,

- the velocity of any unhurried particle is below the absolute speed limit in all reference frames,

- $\quad$ elementary particles of the both kinds may be treated as wave packets.

Thus, in agreement with [2,3], the Galileo's concept [6] may be regarded as an ancestor of the modern relativistic and quantum theories.

\section{CONFLICTS OF INTEREST}

The authors declare no conflicts of interest regarding the publication of this paper.

\section{REFERENCES}

1. Petelin, M. and Thumm, M. (2018) On the Evolution of Approaches to the Space-Time Symmetry. Natural Science, 10, 81-84. https://doi.org/10.4236/ns.2018.103008

2. Mermin, N.D. (1984) Relativity without Light. American Journal of Physics, 52, 119-124. https://doi.org/10.1119/1.13917

3. Feigenbaum, M.J. (2008) The Theory of Relativity-Galileo's Child. The Rockefeller University, New York.

4. Pauli, W. (1921) Theory of Relativity. Encyclopedia of Mathematical Sciences, B.G. Teubner, Leipzig.

5. Feynman, R.P., Leighton, R.B. and Sands, M. (2006) The Feynman Lectures on Physics. Addison-Wesley Longman.

6. Drake, S. (1978) Galileo at Work-His Scientific Biography. University of Chicago Press, Chicago.

7. Petelin, M. (2015) The Universal Speed Limit as an Attribute of the Space-Time Symmetry. Journal of Basic and Applied Physics (JBAP), 4, 8-11.

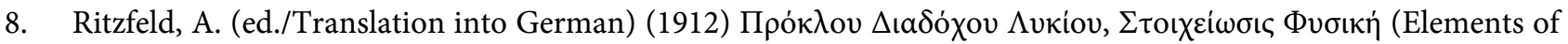
Physics). Teubner, Leipzig.

9. Kimura, W.D. (2017) Electromagnetic Waves and Lasers. Morgan \& Claypool Publishers, San Rafael. https://doi.org/10.1088/978-1-6817-4613-5

10. Kar, D. (2019) Experimental Particle Physics. IOP Publishing Ltd., Bristol. https://doi.org/10.1088/2053-2563/ab1be6

11. Einstein, A. (1905) Ist die Trägheit eines Körpers von seinem Energieinhalt abhängig? Annalen der Physik, 18, 639-643. https://doi.org/10.1002/andp.19053231314

12. Bohm, D. (1951) Quantum Theory. Prentice-Hall, Inc., New York. 